\title{
Sequence Determinants of Bacterial Amyloid Formation
}

\author{
Xuan Wang and Matthew R. Chapman ${ }^{*}$ \\ Department of Molecular, Cellular and Developmental Biology, University of Michigan, $830 \mathrm{~N}$. \\ University, Ann Arbor, MI 48109, U.S.A
}

\section{Summary}

\begin{abstract}
Amyloids are proteinaceous fibers commonly associated with neurodegenerative diseases and prionbased encephalopathies. Many different polypeptides can form amyloid fibers, leading to the suggestion that amyloid is a primitive main-chain-dominated structure. A growing body of evidence suggests that amino acid side chains dramatically influence amyloid formation. The specific role fulfilled by side chains in amyloid formation, especially in vivo, remains poorly understood. Here, we determined the role of internally conserved polar and aromatic residues in promoting amyloidogenesis of the functional amyloid protein, CsgA. CsgA is the major protein component of curli fibers assembled by enteric bacteria such as Escherichia coli and Salmonella spp. In vivo CsgA polymerization into an amyloid fiber requires the $\mathrm{CsgB}$ nucleator protein. The CsgA amyloid core region is composed of five repeating units, defined by regularly spaced Ser, Gln and Asn residues. The results of a comprehensive alanine scan mutagenesis screen showed that Gln and Asn residues at positions 49,54, 139 and 144 were critical for curli assembly. Alanine substitution of Q49 or N144 impeded the ability of $\mathrm{Csg}$ A to respond to $\mathrm{CsgB}$-mediated heteronucleation, and the ability of $\mathrm{Csg} \mathrm{A}$ to self-polymerize in vitro. However, $\mathrm{Csg}$ A proteins harboring these mutations were still seeded by preformed wild-type CsgA fibers in vitro. This suggests that CsgA-fibril-mediated seeding and CsgBmediated heteronucleation have distinguishable mechanisms. Remarkably, Gln residues at positions 49 and 139 could not be replaced by Asn residues without interfering with curli assembly, suggesting that the side chain requirements were especially stringent at these positions. This analysis demonstrates that bacterial amyloid formation is driven by specific side chain contacts and provides a clear illustration of the essential roles of specific side chains in promoting amyloid formation.
\end{abstract}

\section{Keywords}

amyloid; nucleation; polymerization; curli; asparagine and glutamine residues

\section{Introduction}

Amyloid formation is readily associated with neurodegenerative diseases and prion-based encephalopathies. ${ }^{1}$ Amyloid fibers are $4-10 \mathrm{~nm}$ wide, unbranched proteinaceous filaments. ${ }^{1}$ Amyloid fibers possess a characteristic cross- $\beta$ sheet quaternary structure, in which $\beta$ strands are perpendicular to fibril axis. ${ }^{1}$ These fiber structures have specific tinctorial properties, binding to dyes such as Congo red and thioflavin T (ThT). ${ }^{1}$ Amyloid toxicity is complex, but a growing body of work suggests that pre-fiber aggregates are cytotoxic, while mature fibers

\footnotetext{
*Corresponding author. E-mail address: chapmanm@umich.edu.

Publisher's Disclaimer: This is a PDF file of an unedited manuscript that has been accepted for publication. As a service to our customers we are providing this early version of the manuscript. The manuscript will undergo copyediting, typesetting, and review of the resulting proof before it is published in its final citable form. Please note that during the production process errors may be discovered which could affect the content, and all legal disclaimers that apply to the journal pertain.
} 
are relatively benign. ${ }^{2}$ Therefore, understanding the folding cascades involved in amyloid formation is necessary for developing new therapies to target these processes.

A newly described class of 'functional' amyloids suggest that amyloid formation can be an integral part of normal cellular physiology. $3 ; 4$ Functional amyloids provide a unique perspective on amyloidogenesis because the cell must have coevolved mechanisms to prevent the toxicity normally associated with amyloid formation. One of the best understood functional amyloids is curli, a bacterially produced extracellular fiber required for biofilm formation and other community behaviors. ${ }^{5}$ In $E$. coli, the polymerization of the major curli fiber subunit protein CsgA into an amyloid fiber is dependent on the minor curli subunit protein, CsgB. 6 CsgA remains soluble until it encounters outer membrane-localized $\mathrm{CsgB} .{ }^{5} \mathrm{CsgB}$ has also been demonstrated to have amyloid-forming properties and apparently serves as a template for CsgA polymerization. ${ }^{7}$ We previously showed that, like many other amyloids, preformed CsgA fibers could seed soluble CsgA polymerization in vitro. ${ }^{8}$ Therefore, we proposed that the growing CsgA fiber on the cell surface could serves as a template promoting soluble CsgA polymerization in a process akin to seeding. ${ }^{8}$ The molecular details of CsgA fiber-mediated seeding and $\mathrm{CsgB}$-mediated heteronucleation remain poorly described. Because nucleation underlies the rate-limiting step of amyloid propagation, understanding the nature of this mechanism will shed light on how cells control amyloid formation.

The primary sequence of CsgA can be divided into three functional domains: an N-terminal Sec signal sequence (cleaved after translocation into the periplasmic space), an N-terminal 22 amino acid segment (N22) that directs CsgA secretion across the outer membrane, ${ }^{9}$ and an amyloid core region (residues 43 to 151) that contains five imperfect repeating units, each 1923 amino acids in length (Figure 1(a)). ${ }^{5}$ The five repeating units form a protease-resistant structure, ${ }^{10}$ which is proposed to be the amyloid core of CsgA. ${ }^{8}$ These repeats are distinguished by the consensus sequence Ser-X5-Gln-X4-Asn-X5-Gln and are linked by 4 or 5 residues. 10 These Ser, Gln and Asn residues are conserved among CsgA homologs of many enteric bacteria. 8 This high degree of amino acid sequence conservation suggests that these residues may play an important role in curli assembly.

Many polypeptides, if not all, can assemble into an amyloid fiber in vitro even though amyloidforming proteins do not necessarily share amino acid similarities. ${ }^{1}$ Therefore, it has been proposed that amyloid formation is an inherent property of polypeptide main chains. ${ }^{1}$ However, specific residues likely play a role in promoting both disease-associated and functional amyloid formation. Yeast prion protein Sup35p has a Gln/Asn rich domain at Nterminus that has been implicated in prion propagation. 1 ;; $12 ; 13$ Moreover, the specific sequences in this Gln/Asn rich domain govern self-recognition and species-specific seeding activity. ${ }^{14}$ Aromatic residues in the islet amyloid polypeptide fragment positively contribute to its polymerization into amyloid fibers in vitro. ${ }^{15}$ Structural analysis of $A \beta 40$ and $A \beta 42$ revealed that two $\beta$ sheets form a parallel $\beta$-sandwich stabilized by specific intermolecular side chain contacts and changes of these side chains resulted in defective fiber assembly. ${ }^{16 ; 17}$ Therefore, it is clear that side chains can influence amyloid polymerization, but the contribution of side chains to in vivo amyloidogenesis and the exact roles of amino acid side chain contacts remain poorly understood. Here, we performed a comprehensive mutagenesis study on CsgA and identified the residues that promote CsgA amyloidogenesis. We showed that Csg A amyloidogenesis is driven by the side chain contacts of four Gln and Asn residues in N-and $\mathrm{C}$-terminal repeats. These Gln and Asn residues play essential roles in the response to CsgBmediated heteronucleation and the initiation of efficient self-assembly in vitro. Strikingly, these four Gln and Asn residues are not required for CsgA seeding suggesting CsgA seeding and CsgB-mediated heteronucleation have distinct mechanisms. 


\section{Results}

\section{Ala scan mutagenesis of internally conserved polar residues}

The amyloid core of CsgA is composed of five repeating units, defined by internally conserved and regularly spaced Ser, Gln and Asn residues conserved among many enteric bacteria (Figure 1(a)). ${ }^{8}$ We performed an Ala scan mutagenesis on these 20 polar residues to test their importance in directing bacterial amyloid formation. Amyloid formation of each CsgA mutant was initially assessed by growing bacteria on plates amended with Congo red, as cells expressing wild-type CsgA will stain a deep red color on this medium (Figure 1(b)). Only two mutations, Q49A and N144A, significantly reduced Congo red binding relative to wild-type CsgA (Figure 1(b)). Transmission electron microscopy (TEM) was used to validate the Cong red binding phenotypes. $\operatorname{csg} A$ cells (LSR10) transformed with pLR5 (encoding CsgA) produced curli fibers that were indistinguishable from those assembled by wild-type strain MC4100 by TEM. Cells expressing Csg $\mathrm{A}^{\mathrm{Q} 49 \mathrm{~A}}$ or $\mathrm{Csg}^{\mathrm{N} 144 \mathrm{~A}}$ assembled fewer fibers than cells expressing wild-type CsgA observed by TEM (Figure 1(c)).

CsgA polymerization into an amyloid fiber in vivo can also be monitored by its ability to migrate as a monomer on SDS PAGE gels after dissociation by a strong acid, formic acid (FA). 18 For example, CsgA produced by wild-type cells is whole cell-associated and SDS insoluble.

${ }^{19}$ Brief treatment with FA liberates CsgA monomers from curli fibers produced by wild-type strain MC4100. ${ }^{6}$ Similar to the wild-type strain, CsgA produced by csgA/pLR5 was SDS insoluble and required brief pretreatment with FA to migrate into the gel (Figure 2(a), lanes 1 and 2). All of the Ser mutants (S43A, S66A, S88A, S111A and S133A) were also SDS insoluble and associated with the whole cell fraction (Figure 2(a), lanes 5, 6, 13, 14, 21, 22, 29, 30, 37 and 38). However, 13 of 15 Ala substitution mutants of Gln/Asn residues (Q49A, N54A, Q60A, Q72A, N77A, Q94A, N99A, Q105A, Q117A, N122A, Q128A, Q139A and N144A) showed different levels of SDS solubility by whole-cell western analysis, suggesting these polar residues help stabilize the amyloid structure (Figure 2(a)). $\operatorname{CsgA}^{\mathrm{Q} 49 \mathrm{~A}}$ and $\mathrm{CsgA}^{\mathrm{N} 144 \mathrm{~A}}$ were unable to complement Congo red binding to a $\operatorname{csg} A$ mutant (Figure 1(b)), and very little of these mutant proteins could be recovered from whole cell lysates scraped off YESCA plates (Figure 2(a), lanes 7, 8, 41 and 42).

To test the possibility that $\operatorname{Csg} \mathrm{A}^{\mathrm{Q} 49 \mathrm{~A}}$ and $\mathrm{Csg}^{\mathrm{N} 144 \mathrm{~A}}$ were secreted away from the cell as soluble proteins, cells and the underlying agar were collected and analyzed by western blotting. In these samples, called 'plugs', both $\mathrm{CsgA}^{\mathrm{Q} 49 \mathrm{~A}}$ and $\mathrm{Csg} \mathrm{A}^{\mathrm{N} 144 \mathrm{~A}}$ were readily detected and SDS soluble, demonstrating that $\mathrm{Csg}^{\mathrm{Q} 49 \mathrm{~A}}$ and $\mathrm{CsgA}^{\mathrm{N} 144 \mathrm{~A}}$ were stable, secreted to the cell surface and unpolymerized (Figure 2(b), lanes 2, 3, 11 and 12). $\mathrm{CsgA}^{\mathrm{N} 54 \mathrm{~A}}$ and $\mathrm{Csg} \mathrm{A}^{\mathrm{Q} 139 \mathrm{~A}}$ were also significantly different from other mutants in the whole cell SDS solubility assays. $\mathrm{CsgA}^{\mathrm{N} 54 \mathrm{~A}}$ was completely SDS soluble (Figure 2(a), lanes 9 and 10) and $\mathrm{Csg} \mathrm{A}^{\mathrm{Q} 139 \mathrm{~A}}$ was not predominately cell associated (Figure 2(a), lanes 39 and 40). $\operatorname{Csg}^{\mathrm{N} 54 \mathrm{~A}}$ and $\mathrm{Csg} \mathrm{A}^{\mathrm{Q} 139 \mathrm{~A}}$ were SDS soluble detected by western analysis of cells and the underlying agar (Figure 2(b), lanes 4, 5, 8 and 9), suggesting $\operatorname{Csg}^{\mathrm{N} 54 \mathrm{~A}}$ and $\operatorname{Csg} \mathrm{A}^{\mathrm{Q} 139 \mathrm{~A}}$ were not assembled into wild-type like fibers in vivo. Collectively, Q49A and N144A were the most defective mutants in curli formation of the 20 mutants examined. In addition, the N54A and Q139A mutants were also significantly defective in curli assembly as measured by western analysis.

\section{Ala scan mutagenesis of the aromatic residues}

It was reported that aromatic residues may play an important role during amyloidogenesis. ${ }^{15}$; ${ }^{20}$ We tested the contribution of aromatic residues in the CsgA amyloid core region by Ala scan mutagenesis. Except for $\mathrm{CsgA}^{\mathrm{Y151A}}$, Ala substitutions in the aromatic residues resulted in proteins that were phenotypically identical to wild-type CsgA as detected by Congo red binding and whole-cell western analysis (Figure 3(a) and 3(b)). $\operatorname{CsgA}^{\mathrm{Y} 151 \mathrm{~A}}$ was undetectable 
by western analysis of cells and the underlying agar, indicating that Y151A was unstable (Figure 3(c), lanes 5 and 6). When Tyr ${ }^{151}$ was changed to Phe, Trp, Lys or Asp, curli formation was restored as evidenced by Congo red binding and whole-cell western analysis, suggesting Tyr residue at position 151 is not absolutely required (Figure 3(d) and data not shown).

\section{$\mathrm{CsgA}^{\mathrm{Q49A}}$ and $\mathrm{CsgA}^{\mathrm{N144A}}$ are defective in self-polymerization in vitro}

To further characterize the most defective CsgA mutant proteins, we purified $\mathrm{CsgA}^{\mathrm{Q}} 49 \mathrm{~A}$ and $\mathrm{Csg}^{\mathrm{N} 144 \mathrm{~A}}$ and compared their polymerization to wild-type CsgA using the ThT assay described in our previous work. ${ }^{8}$ Wild-type CsgA assembles into an amyloid fiber in vitro at concentrations above $2.0 \mu \mathrm{M}$ in the absence of $\mathrm{CsgB} .{ }^{8}$ Two parameters were used to compare the polymerization kinetics of $\mathrm{Csg} A$ and its mutant analogues. The first kinetic parameter was the time period preceding rapid fiber growth, called lag phase or $\mathrm{T}_{0}$. The second parameter was the time period encompassing the fiber growth phase from initiation of rapid polymerization to its completion, called conversion time $\left(\mathrm{T}_{\mathrm{c}}\right) .{ }^{11} \mathrm{At}$ a concentration of $40 \mu \mathrm{M}$, the $\mathrm{T}_{0}$ of $\operatorname{Csg} \mathrm{A}^{\mathrm{Q} 49 \mathrm{~A}}$ was similar to that ${ }_{\mathrm{N} 144 \mathrm{~A}}$ of $\operatorname{CsgA}$, while the $\mathrm{T}_{\mathrm{c}}$ was much greater than that of CsgA (Figure 4(a)). CsgA polymerization had much greater $\mathrm{T}_{0}$ and $\mathrm{T}_{\mathrm{c}}$ than those of CsgA, suggesting the amido group of Asn at position 144 is critical for aggregation (Figure 4 (a)). After $120 \mathrm{hrs}$, both $\mathrm{Csg}^{\mathrm{Q}} \mathrm{Q}^{4 \mathrm{~A}}$ and $\mathrm{Csg} \mathrm{A}^{\mathrm{N} 144 \mathrm{~A}}$ had assembled into amyloid fibers with similar fiber morphology to wild-type CsgA fibers (Figure 4(b), 4(c) and 4(d)).

\section{CsgA ${ }^{Q 49 A}$ and $\operatorname{Csg} A^{N 144 A}$ are defective in heteronucleation response}

Even though $\operatorname{Csg} \mathrm{A}^{\mathrm{Q} 49 \mathrm{~A}}$ and $\mathrm{Csg} \mathrm{A}^{\mathrm{N} 144 \mathrm{~A}}$ were defective in self-polymerization, in the presence of wild-type CsgA seeds they polymerized with efficiency similar to wild-type CsgA (data not shown). To test of the ability of $\operatorname{Csg} \mathrm{A}^{\mathrm{Q} 49 \mathrm{~A}}$ and $\mathrm{Csg}^{\mathrm{N} 144 \mathrm{~A}}$ to respond to CsgB-mediated heteronucleation, two different approaches were employed. The first was an overlay assay using freshly purified $\mathrm{Csg} \mathrm{A}$ or $\mathrm{Csg} \mathrm{A}$ mutant proteins and cells expressing the $\mathrm{CsgB}$ nucleator protein. ${ }^{21}$ In a CsgB-dependent manner, soluble wild-type CsgA was converted into an amyloid fiber within 1 minute of the overlay as evidenced by Congo red staining, or by TEM. 21 Various concentrations of $\operatorname{Csg} \mathrm{A}, \mathrm{Csg} \mathrm{A}^{\mathrm{Q} 49 \mathrm{~A}}$ and $\mathrm{Csg}^{\mathrm{N} 144 \mathrm{~A}}$ were overlaid on $\operatorname{csg} \mathrm{A}$ cells $\left(\mathrm{CsgB}^{+}\right)$. At a concentration of $10 \mu \mathrm{M}$ or higher, CsgA polymerized into an amyloid fiber in a CsgB-dependent fashion detected by Congo red staining (Figure 5(a)). However, $10 \mu \mathrm{M}$ $\mathrm{CsgA}^{\mathrm{Q} 49 \mathrm{~A}}$ and $\mathrm{Csg}^{\mathrm{N} 144 \mathrm{~A}}$ did not polymerize into amyloid-like fibers under the same conditions (Figure 5(a)). When the concentration of Csg $\mathrm{A}^{\mathrm{Q} 49 \mathrm{~A}}$ and $\mathrm{Csg}^{\mathrm{N} 144 \mathrm{~A}}$ was increased to $40 \mu \mathrm{M}$, a Congo red-binding structure on the surface of CsgB-expressing cells was detected (Figure 5(a)).

Curli fibers are proposed to assemble after CsgA is secreted to the extracellular space. This can be illustrated by interbacterial complementation where a $\operatorname{csgA}$ mutant strain $\left(\mathrm{CsgB}^{+}\right)$is grown in close proximity to a $\operatorname{csg} B$ mutant strain $\left(\mathrm{CsgA}^{+}\right)$. CsgA molecules secreted by $\operatorname{csg} B$ donor cells polymerize into amyloid fibers on the $\operatorname{csg} A$ acceptor cells, as shown in Figure 5(b). ${ }^{6}$ We performed an interbacterial complementation assay to test CsgB-mediated heteronucleation responsiveness of $\mathrm{Csg} \mathrm{A}, \mathrm{Csg} \mathrm{A}^{\mathrm{Q} 49 \mathrm{~A}}$ and $\mathrm{Csg} \mathrm{A}^{\mathrm{N} 144 \mathrm{~A}}$ in vivo. Csg A secreted from $\operatorname{csg} B A / \mathrm{pLR} 5$ (donor) responded to the heteronucleation of CsgB on the $\operatorname{csg} A$ cells (acceptor), and formed an amyloid fiber on the surface of $\operatorname{csg} A$ cells as demonstrated by Congo red (Figure 5(b)). ${ }^{6}$ There was reduced Congo red binding on the surface of $\operatorname{csg} A$ cells when $\operatorname{csg} B A$ cells containing plasmid encoding $\operatorname{Csg} \mathrm{A}^{\mathrm{Q} 49 \mathrm{~A}}$ or $\operatorname{Csg} \mathrm{A}^{\mathrm{N} 144 \mathrm{~A}}$ were grown adjacently to $\operatorname{csg} A$ cells (Figure 5(b)). The protein level of $\operatorname{Csg} \mathrm{A}, \operatorname{Csg} \mathrm{A}^{\mathrm{Q} 49 \mathrm{~A}}$ and $\operatorname{Csg} \mathrm{A}^{\mathrm{N} 144 \mathrm{~A}}$ produced in $\operatorname{csg} B A$ genetic background is similar as detected by western analysis of cells and the underlying agar (data not shown). These data collectively demonstrate that the polymerization of $\operatorname{Csg} \mathrm{A}^{\mathrm{Q} 49 \mathrm{~A}}$ and $\operatorname{Csg} \mathrm{A}^{\mathrm{N} 144 \mathrm{~A}}$ were defective in responding to the heteronucleator, CsgB heterogeneous nucleator $\mathrm{CsgB}$. 


\section{Ala substitutions of Q49, N54, Q139 and N144 result in the dramatic loss of heteronucleation response to CsgB}

In order to abolish the four critical Gln/Asn residues identified by our Ala scan mutagenesis, we constructed the mutant $\mathrm{Q} 49 \mathrm{~A} / \mathrm{N} 54 \mathrm{~A} / \mathrm{Q} 139 \mathrm{~A} / \mathrm{N} 144 \mathrm{~A}$. In vivo

$\mathrm{Csg}^{\mathrm{Q} 49 \mathrm{~A} / \mathrm{N} 54 \mathrm{~A} / \mathrm{Q} 139 \mathrm{~A} / \mathrm{N} 144 \mathrm{~A}}$ (Csg $\mathrm{A}^{\text {slowgo }}$ ) completely lost the ability to assemble into amyloid fibers as detected by Congo red binding, TEM and whole-cell western analysis (data not shown). We tested the competence of Csg $\mathrm{A}^{\text {slowgo }}$ to respond to CsgB-mediated heteronucleation by overlay assay. Purified homogeneous $\mathrm{Csg}^{\text {slowgo }}$ could not be promoted to form amyloid fibers by cell-associated $\mathrm{CsgB}$ as evidenced by the lack of Congo red binding in the overlay assay even at a concentration of $100 \mu \mathrm{M}$ (Figure 6(a)). There was no Congo red binding on the surface of $\operatorname{csg} A$ cells when $\operatorname{csg} B A$ cells containing plasmid encoding CsgA $\mathrm{A}^{\text {slowgo }}$ were grown adjacently to $\operatorname{csg} A$ cells shown in Figure 6(b), suggesting that


interbacterial complementation. The protein level of $\mathrm{Csg}^{\text {slowgo }}$ was similar to wild-type CsgA in this genetic background (data not shown). Collectively, these results demonstrate that $\mathrm{Csg}^{\text {slowgo }}$ remains unpolymerized even in the presence of the heteronucleator CsgB, suggesting these four polar residues are required in $\mathrm{CsgB}$-mediated heteronucleation.

\section{Q49, N54, Q139 and N144 are required for efficient self-polymerization and CsgB trunc $_{\text {cross- }}$ seeding not for CsgA seeding}

$\mathrm{Csg}^{\text {slowgo }}$ was purified and its polymerization kinetics were analyzed in vitro. The $\mathrm{T}_{0}$ and $\mathrm{T}_{\mathrm{c}}$ of Csg A $^{\text {slowgo }}$ were much higher than CsgA (Figure 7(a)), suggesting that Q49, N54, Q139 and N144 play critical roles in self-polymerization in vitro. Self-assembled CsgA ${ }^{\text {slowgo }}$ fibers were similar to CsgA fibers shown by TEM (Figure 7(b)). Interestingly, wild-type Csg A seeds efficiently eliminated the lag phase of the polymerization of $\mathrm{Csg}^{\text {slowgo }}$ as detected by $\mathrm{ThT}$ assay (Figure 7(c)). In the presence of CsgA seeds, the polymerization rate Csg $\mathrm{A}^{\text {slowgo }}$ is very similar to wild-type $\operatorname{CsgA}{ }^{8}$ and ThT signal reached stationary phase within 5 hours (Figure 7 (c)). Polymerized Csg $\mathrm{A}^{\text {slowgo }}$ fibers promoted by wild-type CsgA were similar to CsgA fibers shown by TEM (Figure 7(d)). A nucleation-competent CsgB truncation mutant (called $\mathrm{CsgB}_{\text {trunc }}$ ) was purified as previously described. ${ }^{7} \mathrm{CsgB}_{\text {trunc }}$ has been demonstrated to efficiently seed the polymerization of wild-type CsgA. ${ }^{7}$ Unlike CsgA seeds, $C_{s g} B_{\text {trunc }}$ seeds did not efficiently promote the polymerization of $\mathrm{Csg}^{\text {slowgo }}$ under the same condition shown

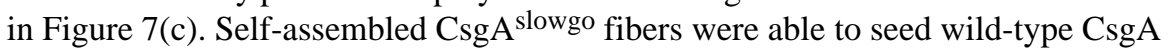
polymerization, suggesting $\mathrm{Csg}^{\text {slowgo }}$ fibers are similar to wild-type CsgA fibers in terms of seeding specificity (data not shown).

\section{Conservative $\mathrm{Q} / \mathrm{N}$ substitutions}

It was proposed that in vitro amyloid formation is strongly influenced by simple physicochemical factors of polypeptides such as hydrophobicity, charge and secondary structure propensity. $1 ; 22$ Ala substitutions at positions 49, 54, 139 and 144 decreased CsgA polymerization in vivo. To test the stringency of the amino acid present at these critical positions, we constructed four mutants (Q49N, N54Q, Q139N and N144Q) that contained relatively conservative amino acid changes. Gln and Asn residues have similar physicochemical features, including the same amido group. Surprisingly, even though the side chain of Asn is only one carbon distance shorter than that of Gln, Csg A ${ }^{\mathrm{Q}}{ }^{4 N}$ had dramatically reduced Congo red binding and was almost undetectable in lysates of whole cells scraped off YESCA plate (Figure 8(a) and 8(b), lanes 7 and 8). CsgA ${ }^{\mathrm{Q} 49 \mathrm{~N}}$ was stable and secreted into the agar as detected by western analysis of cells and the underlying agar (Figure 8(c), lanes 7 and 8). In addition, whole-cell western analysis showed that Q139N (Figure 8(b), lanes 15 and 16) was as defective as Q139A (Figure 2(a), lanes 39 and 40). The sequence requirements at positions 49 and 139 appear extremely stringent during fiber assembly since either Ala or Asn 
was not tolerated at these positions. The Congo red binding of $\mathrm{CsgA}^{\mathrm{N} 144 \mathrm{Q}}$ and $\mathrm{Csg} \mathrm{A}^{\mathrm{N} 54 \mathrm{Q}}$ was similar to that of wild-type $\operatorname{Csg} A$ (Figure $8(a)$ ). In addition, $C \operatorname{sg} \mathrm{A}^{\mathrm{N} 144 \mathrm{Q}}$ and $\mathrm{Csg} \mathrm{A}^{\mathrm{N} 54 \mathrm{Q}}$ were predominately SDS insoluble as shown by whole-cell western analysis (Figure 8(b), lanes 11, 12,13 and 14). The SDS insolubility of $\operatorname{Csg}^{\mathrm{N} 144 \mathrm{Q}}$ and $\mathrm{Csg}^{\mathrm{N} 54 \mathrm{Q}}$ was similar to wild-type CsgA as detected by western analysis of cells and the underlying agar (Figure 8(c), lanes 11, 12,15 and 16). We observed wild-type like fibers assembled by the $\operatorname{csg} A$ strain that contained plasmid encoding Csg $\mathrm{A}^{\mathrm{N} 144 \mathrm{Q}}$ by TEM, while relatively few fibers were observed on the $\operatorname{csg} A$ strain expressing $\operatorname{Csg} \mathrm{A}^{\mathrm{Q} 49 \mathrm{~N}}$ (Figure 8(d)). Collectively, in vivo Gln residues at positions 49 and 139 could not be replaced by Asn, while Asn residues at position 54 and 144 could be replaced by Gln and function like wild-type CsgA. In vitro $\mathrm{Csg} \mathrm{A}^{\mathrm{Q} 49 \mathrm{~N}}$ was as defective as $\mathrm{CsgA}^{\mathrm{Q} 49 \mathrm{~A}}$ in both heteronucleation responsiveness and self-polymerization. $\mathrm{Csg}^{\mathrm{Q}} \mathrm{Q}^{\mathrm{N}} \mathrm{N}$ polymerization had greater $\mathrm{T}_{\mathrm{c}}$ than that of $\mathrm{Csg} \mathrm{A}$ at the same concentration measured by ThT assay (Figure 8(e)). Csga $\mathrm{A}^{\mathrm{Q}} 9 \mathrm{~N}$ did not respond to $\mathrm{CsgB}$ nucleation at $10 \mu \mathrm{M}$ as detected by overlay assay (Figure 8(e) insert). It has been proposed that amino acid content can be an important factor in promoting amyloid formation. ${ }^{23 ;} 24$ To test whether N144Q can suppress the polymerization defect of Q49N, we constructed the mutant Q49N/N144Q. Like Q49N, Q49N/N144Q had significantly reduced Congo red binding relative to wild-type CsgA (Figure 8(f)). Collectively, these findings suggest that CsgB-mediated heteronucleation and CsgA selfpolymerization require spatially constrained contacts between certain amino acid side chains.

\section{Discussion}

Curli function as part of the extracellular matrix produced by many Gram-negative enteric bacteria. Curli assembly is a precisely coordinated process that is highly amenable to study because of the sophisticated genetic and biochemical tools afforded by E. coli. Here, we elucidated the CsgA sequence determinants that drive amyloid formation. We found that Gln and Asn residues at the $\mathrm{N}$ - and $\mathrm{C}$ - terminal repeats are critical for curli assembly. These Gln and Asn residues are necessary for $\mathrm{Csg} \mathrm{A}$ to respond to $\mathrm{CsgB}$-mediated heteronucleation and to initiate efficient CsgA self-assembly in vitro.

\section{The contribution of amino acid side chains to functional amyloid formation}

Unlike amorphous aggregates formed by nonspecific hydrophobic interactions, amyloid fibers are highly ordered proteinaceous polymers that are stabilized by specific interactions. The side chains can clearly influence amyloidogenesis. ${ }^{1}$ But how specific sequences influence amyloid formation in vivo remains poorly understood. We demonstrated that four internally conserved Gln and Asn residues were required to respond to CsgB-mediated heteronucleation. When these four residues are changed to Ala, the resulting protein, $\mathrm{Csg}^{\text {slowgo }}$, cannot efficiently initiate self-assembly in vitro as indicated by an extremely long lag phase before polymerization (Figure 7(a)). CsgA ${ }^{\text {slowgo }}$ also cannot efficiently respond to CsgB-mediated heteronucleation (Figure 6). These results suggest that the specific side chain interactions are the driving forces to efficiently initiate bacterial amyloid formation in vivo and in vitro.

Interestingly, we found that these Gln/Asn residues required for CsgB-mediated heteronucleation are not necessary for CsgA fiber-mediated seeding, suggesting that $\mathrm{Csg} \mathrm{A}$ seeding and $\mathrm{CsgB}$-mediated heteronucleation may have somewhat distinct mechanisms (Figure 7(c)). Csg $\mathrm{A}^{\text {slowgo }}$ is completely defective in amyloid formation in vivo, although it can inefficiently assemble into amyloid fibers in vitro (Figure 7(a) and 7(b)). This distinction between in vitro and in vivo behaviors suggests that in vivo amyloid formation has highly stringent requirements that limit initiation of amyloid formation to CsgB-mediated heteronucleation. For example, CsgA remains soluble in the extracellular milieu in the absence of nucleator $\mathrm{CsgB}$ even though CsgA is amyloidogenic in vitro. ${ }^{8}$ This indicates that CsgA may diffuse into the extracellular milieu if it cannot participate in efficient nucleation. Curli also provides an elegant example how amyloidogenecity can be temporally and spatially controlled. 
It is possible that an evolved functional amyloid such as curli capitalizes on specific side-chain interactions to tightly regulate and control amyloidogenecity.

\section{The diverse sequence determinants of amyloid formation}

The sequence-specific determinants of amyloid formation have been most studied in vitro. Clearly, different amyloid proteins employ various interactions such as hydrogen bonding, hydrophobic and electrostatic interactions to promote intra- and inter-molecular associations. For example, the hydrophobic stretches in $\mathrm{A} \beta 42$, rather than specific side chains, are sufficient to promote aggregation. ${ }^{25}$ Yeast prion proteins Sup35p, Ure2p and Rnq1p all have Gln/Asn rich domains that are essential for prion propagation in vivo and in vitro. $11 ; 26 ; 27 ; 28 \mathrm{In}$ Huntington's disease, polyglutamine peptides play an essential role in molecular etiology. In addition, the length of polyglutamine peptides is positively correlated to in vitro aggregation. ${ }^{29} \mathrm{Gln} /$ Asn rich domains are proposed to have a high potential to be amyloidogenic because they can form intramolecular hydrogen bonds. ${ }^{26} \mathrm{Here}$, we showed that the internally conserved Gln and Asn residues at the $\mathrm{N}$ - and $\mathrm{C}$ - terminal repeats are critical for CsgA fiber formation. The initiation of CsgA polymerization both in vitro and in vivo appears to be dependent on hydrogen bonds formed by these Gln and Asn residues.

Moreover, we showed that 13 of 15 Ala substitution mutants of Gln/Asn residues (Q49A, N54A, Q60A, Q72A, N77A, Q94A, N99A, Q105A, Q117A, N122A, Q128A, Q139A and N144A) showed somewhat more sensitive to SDS relative to wild-type CsgA (Figure 2(a)), suggesting these polar residues help stabilize the amyloid structure. Interestingly, the polar amides of these internally conserved Gln and Asn residues were proposed to form hydrogen bonds with backbones to stabilize its fibril structure in a CsgA predicted structural model. ${ }^{10}$ It is possible that these critical Gln/Asn residues not only play an important role to initiate fiber assembly but also stabilize the fiber structure when fibers are formed.

It was proposed that interactions among aromatic residues by $\pi$-stacking possibly play a critical role in self-assembly of some amyloid fibrils such as islet amyloid polypeptide. ${ }^{15}$ To assess the role of aromatic residues in guiding in vivo CsgA amyloidogenesis, we individually replaced each aromatic residue in the amyloid core of CsgA, revealing that aromatic residues do not significantly contribute to curli assembly (Figure 3).

\section{Bacterial amyloid formation is influenced by the spatial arrangement of $Q / N$ residues}

It has been suggested that amyloid formation is determined by simple physicochemical factors of polypeptides such as hydrophobicity, charge and secondary structure propensity. ${ }^{22}$ Furthermore, the amyloidogenecity of yeast prions Ure2p and Sup35p appears to be encoded by a particular amino acid composition, rather than specific sequences. ${ }^{23 ;} 24$ In contrast, our work demonstrates that CsgA amyloidogenesis, especially in vivo, is highly dependent on its specific sequence rather than on its overall amino acid composition or the physicochemical properties of polypeptide chains. The Gln residue at positions 49 and 139 of CsgA could not be replaced by Asn residues without interfering with curli assembly (Figure 8), even though Asn shares many physicochemical features with Gln. CsgA ${ }^{4} 49 \mathrm{~N}-\mathrm{N} 144 \mathrm{Q}$ and wild-type CsgA share an identical composition of amino acids, but Csg $\mathrm{A}^{\mathrm{Q} 49 \mathrm{~N}-\mathrm{N} 144 \mathrm{Q}}$ is dramatically defective in curli assembly (Figure 8(f)). Additionally, although CsgA ${ }^{\mathrm{Q} 49 \mathrm{~A}}, \mathrm{CsgA}^{\mathrm{Q} 60 \mathrm{~A}}, \mathrm{CsgA}^{\mathrm{Q} 94 \mathrm{~A}}$, $\mathrm{Csg}^{\mathrm{Q} 105 \mathrm{~A}}, \mathrm{Csg}^{\mathrm{Q} 117 \mathrm{~A}}, \mathrm{Csg}^{\mathrm{Q}}{ }^{\mathrm{128A}}, \mathrm{Csg}^{\mathrm{Q}}{ }^{150 \mathrm{~A}}$ have the identical composition of amino acids, $\mathrm{Csg} \mathrm{A}^{\mathrm{Q} 49 \mathrm{~A}}$ is dramatically defective than other Ala mutants in fiber assembly (Figure 1 (b) and 2(a)). We previously showed that $\mathrm{N}$ - and $\mathrm{C}$-terminal $\mathrm{Csg} \mathrm{A}$ repeats drive curli assembly. 21 Our current study clearly suggests the position of Gln or Asn residues plays an equally important role for amyloid assembly. 
Globular protein folding depends on specific side chain contacts. Whether amyloid formation, especially in vivo, depends on specific side chain contacts is just beginning to be tested. Our work demonstrates that the initiation of $\mathrm{Csg} A$ amyloid formation requires specific amino acid side chains. Specific side chain contacts are likely to facilitate the spatial and temporal control over the polymerization of functional amyloids.

\section{Materials and Methods \\ Bacterial Growth}

To induce curli production, bacteria were grown on YESCA plates $(1.0 \mathrm{~g}$ yeast extract, $10 \mathrm{~g}$ casamino acids and $20 \mathrm{~g}$ agar per liter) at $26^{\circ} \mathrm{C}$ for $48 \mathrm{~h} .{ }^{18}$ Antibiotics were added to plates at the following concentrations: kanamycin $50 \mu \mathrm{g} / \mathrm{ml}$, chloramphenicol $25 \mu \mathrm{g} / \mathrm{ml}$, or ampicillin $100 \mu \mathrm{g} / \mathrm{ml}$. Curli production was monitored by using Congo red-YESCA (CR-YESCA) plate. 18

\section{Strains and Plasmids}

Strains LSR10 (MC4100::csgA), LSR12 (C600::csgBAC and csgDEFG), LSR13 (MC4100::csgBA) and MHR261 (MC4100::csgB) and plasmids pLR5 (encoding CsgA), pLR2 (vector control) and pMC1(encoding $\mathrm{CsgG}$ ) were previously constructed. $6 ; 7 ; 9 ; 18$ Plasmids containing CsgA mutations were constructed by site-specific mutagenesis using standard overlapping PCR extension. PCR products containing relevant mutations and NcoI/BamHI restriction endonuclease sites at 5'/3' ends were cloned downstream of the $\operatorname{csg} B A$ promoter into $\mathrm{NcoI} / \mathrm{BamHI}$ sites of vector pLR2. The expression vector pMC3 was generated previously.

18 To express and purify CsgA mutant proteins, PCR amplified mutant sequences including C-terminal hexahistidine tag were cloned into pMC3, replacing the sequence encoding CsgAhis. Sequences of constructs were verified by DNA sequencing.

\section{Western analysis}

The immunoblotting of CsgA in whole-cells and plugs was performed as previously described. 18 Briefly, bacterial cells grown on YESCA plate for $48 \mathrm{~h}$ were scraped off and normalized by optical density at $600 \mathrm{~nm}$. Cell pellets were resuspended in 2X SDS loading buffer either with or without prior formic acid (FA) treatment as previously described. ${ }^{18}$ Alternatively, 8 mm circular plugs including cells and underlying agar were resuspended in 2X SDS loading buffer either with or without prior FA treatment. Samples were electrophoresed on $15 \%$ sodium dodecyl sulfate (SDS)-polyacrylamide and transferred onto polyvinylidene difluoride membrane using standard techniques. Western blots were probed and developed as previously described. 7

\section{Transmission Electron Microscopy}

A Philips CM10 Transmission Electron Microscope was used to visualize the cell samples and protein fiber aggregates. Samples $(10 \mu \mathrm{l})$ were placed on Formvar-coated copper grids (Ernest F. Fullam, Inc., Latham, NY) for 2 min, washed with deionized water, and negatively stained with $2 \%$ uranyl acetate for $90 \mathrm{sec}$.

\section{In vitro polymerization and nucleation assay}

CsgA or CsgA mutant proteins and $\mathrm{CsgG}$ were co-overexpressed by induction with isopropyl $\beta$-D-1-thiogalactopyranoside (IPTG) in LSR 12 (C600::csgBAC and $c s g D E F G$ ) strain. CsgA or CsgA mutant proteins were secreted to the supernatant and purified as previously described. 8 After removal of imidazole by a desalting column, purified proteins were passed through a $30 \mathrm{kD}$ cutoff filter (Amicon® Ultra, MA) to remove possible aggregates or seeds that might alter polymerization kinetics. Purified homogeneous proteins were loaded on 96-well opaque 
plate and ThT was added to a concentration of $20 \mu \mathrm{M}$. ThT fluorescence was measured every 10 min after shaking $5 \mathrm{sec}$ by a Spectramax M2 plate reader (Molecular Devices, Sunnyvale, CA) set to $438 \mathrm{~nm}$ excitation and $495 \mathrm{~nm}$ emission with a $475 \mathrm{~nm}$ cutoff. Alternatively, samples were kept at room temperature in the presence of $0.02 \% \mathrm{NaN}_{3}$. At the indicated times, aliquots were removed, ThT was added at a concentration of $20 \mu \mathrm{M}$ and fluorescence intensity was measured as described above. For seeding reactions, CsgA fiber seeds, prepared as previously described, ${ }^{8}$ were added to freshly purified CsgA mutant proteins immediately before the start of ThT fluorescence assay.

\section{Overlay assay}

$10 \mu$ freshly purified proteins were spotted onto a lawn of $\operatorname{csg} A$ (LSR10) or $\operatorname{csg} B A$ (LSR13) cells grown on YESCA plates at $26^{\circ} \mathrm{C}$ for $48 \mathrm{~h}$. Samples were incubated on cells for $10 \mathrm{~min}$ at room temperature, stained with a $0.5 \mathrm{mg} / \mathrm{ml}$ Congo red in $50 \mathrm{mM}$ potassium phosphate $(\mathrm{pH}$ 7.2) for $5 \mathrm{~min}$, then washed with potassium phosphate buffer and photographed.

\section{Interbacterial complementation}

Cells that secrete CsgA or CsgA mutant proteins were streaked adjacently to $\operatorname{csg} A$ cells on a CR-YESCA plate. The plates were photographed after $48 \mathrm{~h}$ growth at $26^{\circ} \mathrm{C}$.

\section{Acknowledgements}

We would like to thank members of the Chapman laboratory and Ryan Frisch forhelpful discussions and review of this manuscript. We thank Bryan McGuffie for his technical help in the preparation of figures. This work was supported by NIH award AI073847-01.

\section{Abbreviations used are}

\section{ThT}

thioflavin $\mathrm{T}$

FA

formic acid

TEM

Transmission Electron Microscopy

\section{References}

1. Chiti F, Dobson CM. Protein misfolding, functional amyloid, and human disease. Annu Rev Biochem 2006;75:333-66. [PubMed: 16756495]

2. Hardy J, Selkoe DJ. The amyloid hypothesis of Alzheimer's disease: progress and problems on the road to therapeutics. Science 2002;297:353-6. [PubMed: 12130773]

3. Fowler DM, Koulov AV, Balch WE, Kelly JW. Functional amyloid--from bacteria to humans. Trends Biochem Sci 2007;32:217-24. [PubMed: 17412596]

4. Hammer ND, Wang X, McGuffie BA, Chapman MR. Amyloids: Friend or Foe? J Alzheimers Dis. 2008(in press)

5. Barnhart MM, Chapman MR. Curli biogenesis and function. Annu Rev Microbiol 2006;60:131-47. [PubMed: 16704339]

6. Hammar M, Bian Z, Normark S. Nucleator-dependent intercellular assembly of adhesive curli organelles in Escherichia coli. Proc Natl Acad Sci U S A 1996;93:6562-6. [PubMed: 8692856]

7. Hammer ND, Schmidt JC, Chapman MR. The curli nucleator protein, CsgB, contains an amyloidogenic domain that directs CsgA polymerization. Proc Natl Acad Sci U S A. 2007

8. Wang X, Smith DR, Jones JW, Chapman MR. In vitro polymerization of a functional Escherichia coli amyloid protein. J Biol Chem 2007;282:3713-9. [PubMed: 17164238] 
9. Robinson LS, Ashman EM, Hultgren SJ, Chapman MR. Secretion of curli fibre subunits is mediated by the outer membrane-localized CsgG protein. Mol Microbiol 2006;59:870-81. [PubMed: 16420357]

10. Collinson SK, Parker JM, Hodges RS, Kay WW. Structural predictions of AgfA, the insoluble fimbrial subunit of Salmonella thin aggregative fimbriae. J Mol Biol 1999;290:741-56. [PubMed: 10395827]

11. DePace AH, Santoso A, Hillner P, Weissman JS. A critical role for amino-terminal glutamine/ asparagine repeats in the formation and propagation of a yeast prion. Cell 1998;93:1241-52. [PubMed: 9657156]

12. Osherovich LZ, Cox BS, Tuite MF, Weissman JS. Dissection and design of yeast prions. PLoS Biol 2004;2:E86. [PubMed: 15045026]

13. Liu JJ, Lindquist S. Oligopeptide-repeat expansions modulate 'protein-only' inheritance in yeast. Nature 1999;400:573-6. [PubMed: 10448860]

14. Tessier PM, Lindquist S. Prion recognition elements govern nucleation, strain specificity and species barriers. Nature 2007;447:556-61. [PubMed: 17495929]

15. Gazit E. A possible role for pi-stacking in the self-assembly of amyloid fibrils. Faseb J 2002;16:7783. [PubMed: 11772939]

16. Petkova AT, Ishii Y, Balbach JJ, Antzutkin ON, Leapman RD, Delaglio F, Tycko R. A structural model for Alzheimer's beta -amyloid fibrils based on experimental constraints from solid state NMR. Proc Natl Acad Sci U S A 2002;99:16742-7. [PubMed: 12481027]

17. Luhrs T, Ritter C, Adrian M, Riek-Loher D, Bohrmann B, Dobeli H, Schubert D, Riek R. 3D structure of Alzheimer's amyloid-beta(1-42) fibrils. Proc Natl Acad Sci U S A 2005;102:17342-7. [PubMed: 16293696]

18. Chapman MR, Robinson LS, Pinkner JS, Roth R, Heuser J, Hammar M, Normark S, Hultgren SJ. Role of Escherichia coli curli operons in directing amyloid fiber formation. Science 2002;295:8515. [PubMed: 11823641]

19. Collinson SK, Emody L, Muller KH, Trust TJ, Kay WW. Purification and characterization of thin, aggregative fimbriae from Salmonella enteritidis. J Bacteriol 1991;173:4773-81. [PubMed: 1677357]

20. Azriel R, Gazit E. Analysis of the minimal amyloid-forming fragment of the islet amyloid polypeptide. An experimental support for the key role of the phenylalanine residue in amyloid formation. J Biol Chem 2001;276:34156-61. [PubMed: 11445568]

21. Wang X, Hammer ND, Chapman MR. The molecular basis of bacterial amyloid polymerization and nucleation. 2008Submitted

22. Chiti F, Stefani M, Taddei N, Ramponi G, Dobson CM. Rationalization of the effects of mutations on peptide and protein aggregation rates. Nature 2003;424:805-8. [PubMed: 12917692]

23. Ross ED, Edskes HK, Terry MJ, Wickner RB. Primary sequence independence for prion formation. Proc Natl Acad Sci U S A 2005;102:12825-30. [PubMed: 16123127]

24. Ross ED, Baxa U, Wickner RB. Scrambled prion domains form prions and amyloid. Mol Cell Biol 2004;24:7206-13. [PubMed: 15282319]

25. Kim W, Hecht MH. Generic hydrophobic residues are sufficient to promote aggregation of the Alzheimer's Abeta42 peptide. Proc Natl Acad Sci U S A 2006;103:15824-9. [PubMed: 17038501]

26. Michelitsch MD, Weissman JS. A census of glutamine/asparagine-rich regions: implications for their conserved function and the prediction of novel prions. Proc Natl Acad Sci U S A 2000;97:11910-5. [PubMed: 11050225]

27. Sondheimer N, Lindquist S. Rnq1: an epigenetic modifier of protein function in yeast. Mol Cell 2000;5:163-72. [PubMed: 10678178]

28. Taylor KL, Cheng N, Williams RW, Steven AC, Wickner RB. Prion domain initiation of amyloid formation in vitro from native Ure2p. Science 1999;283:1339-43. [PubMed: 10037606]

29. Chen S, Ferrone FA, Wetzel R. Huntington's disease age-of-onset linked to polyglutamine aggregation nucleation. Proc Natl Acad Sci U S A 2002;99:11884-9. [PubMed: 12186976] 
(a)

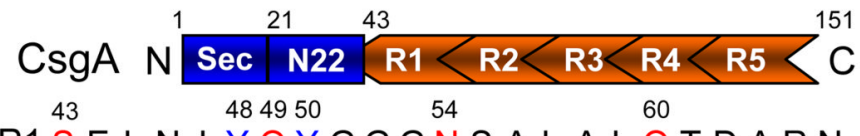

R1SELN I YQYGGGNSALALQTDARN R2 $S_{88}^{66} D T$ I T ${ }_{94}^{72} H G G_{97} \prod_{99} G A D V G \underset{105106}{83}$ D D

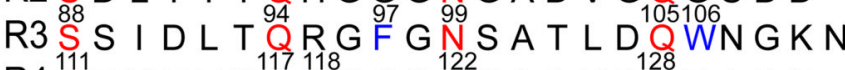
R4 S $S M T V K Q F G G G N N_{139}^{11}$ A A V D Q TAS N $R 5 S^{133} S \vee N \vee T{ }^{139} \vee{ }^{142} F N^{144} N A T A H Q Y^{151}$

(b)

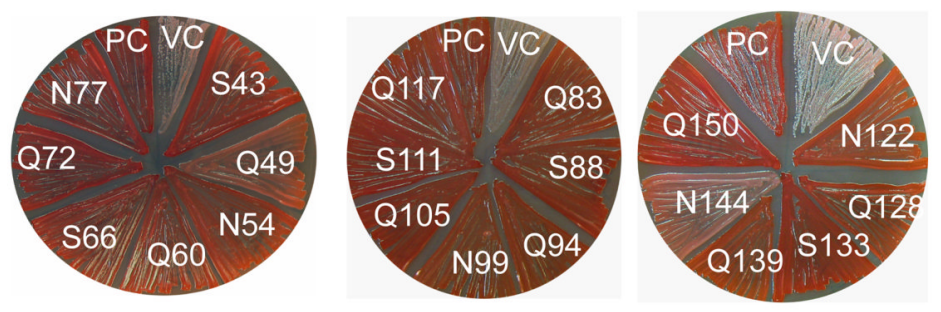

(c)
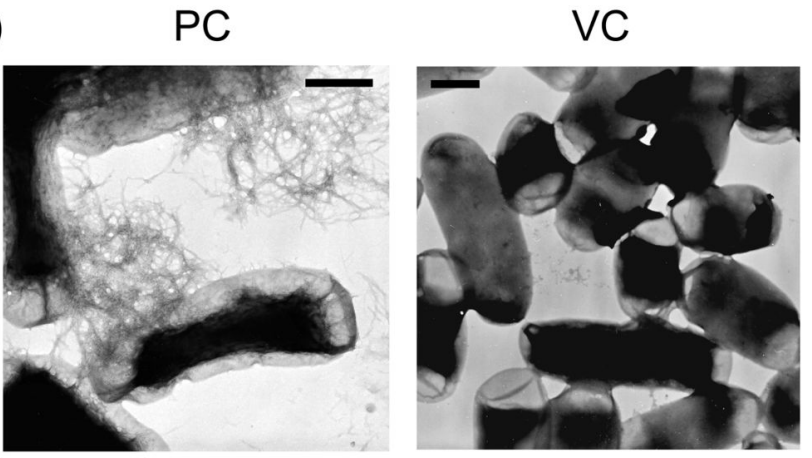

Q49A

$\mathrm{N} 144 \mathrm{~A}$
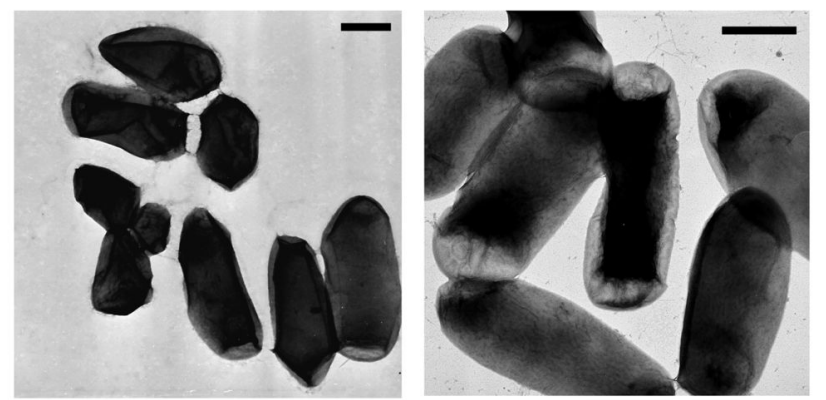

Figure 1. Effect of Ala substitutions of conserved Ser, Gln and Asn of CsgA on curli assembly (a) Schematic of CsgA including an N-terminal Sec signal sequence and the N-terminal 22 residues that precede the five repeating units. The regularly-spaced Ser, Gln and Asn residues are indicated in red color and the aromatic residues are indicated in blue color. The position of highlighted residues is indicated above the residue. (b) CR-YESCA plate with $\operatorname{csg} A$ cells transformed with the plasmids encoding CsgA with Ala substitution after $48 \mathrm{~h}$ of growth at $26^{\circ} \mathrm{C}$. $\operatorname{csg} A / \mathrm{pLR} 5$ and $\operatorname{csg} A / \mathrm{pLR} 2$ were used as the positive control (PC) and vector control (VC), respectively. The specific Ala substitution is indicated. (c) Negative-stain TEM micrographs of $\operatorname{csg} A$ cells containing vector control (pLR2) or plasmids encoding wild-type CsgA (pLR5), CsgA ${ }^{\mathrm{Q} 49 \mathrm{~A}}$ and CsgA ${ }^{\mathrm{N} 144 \mathrm{~A}}$. Scale bars are equal to $500 \mathrm{~nm}$. 
(a)
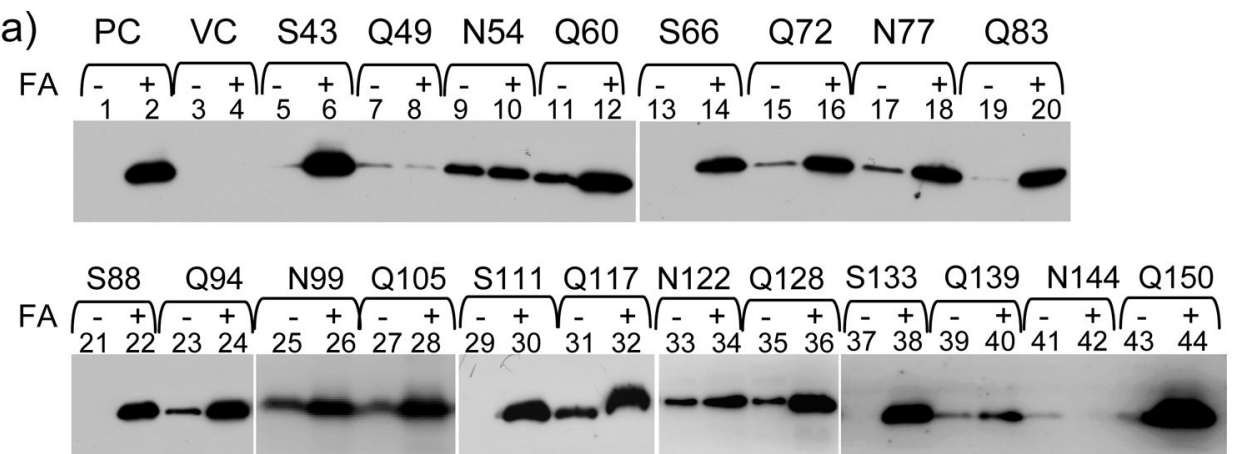

(b)

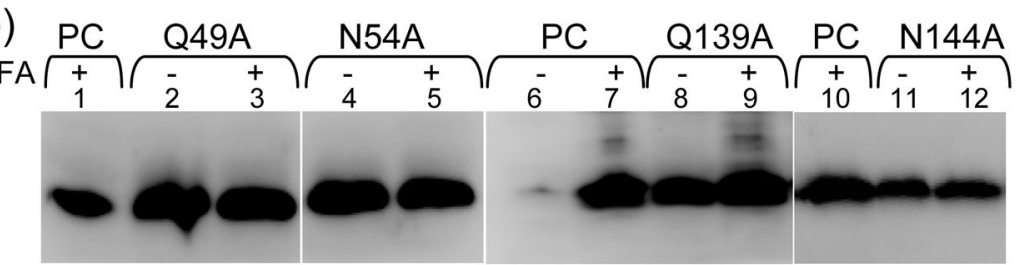

Figure 2. Western analysis of CsgA mutants with Ala substitutions of internally conserved Ser, GIn and Asn

(a) Western blots of whole-cell lysates from $\operatorname{csg} A$ cells containing vector control or plasmids encoding wild-type CsgA or indicated Ala substituted mutants. (b) Western blot of whole-cells and underlying agar (agar plugs) from $\operatorname{csg} A$ strains containing plasmids encoding CsgA, $\mathrm{CsgA}^{\mathrm{Q} 49 \mathrm{~A}}, \mathrm{CsgA}^{\mathrm{N} 54 \mathrm{~A}}, \mathrm{CsgA}^{\mathrm{Q} 139 \mathrm{~A}}$ or $\mathrm{CsgA}^{\mathrm{N} 144 \mathrm{~A}}$. Samples were treated with (+) or without (-) FA. The blots were probed with anti-CsgA antibody. 
(a)

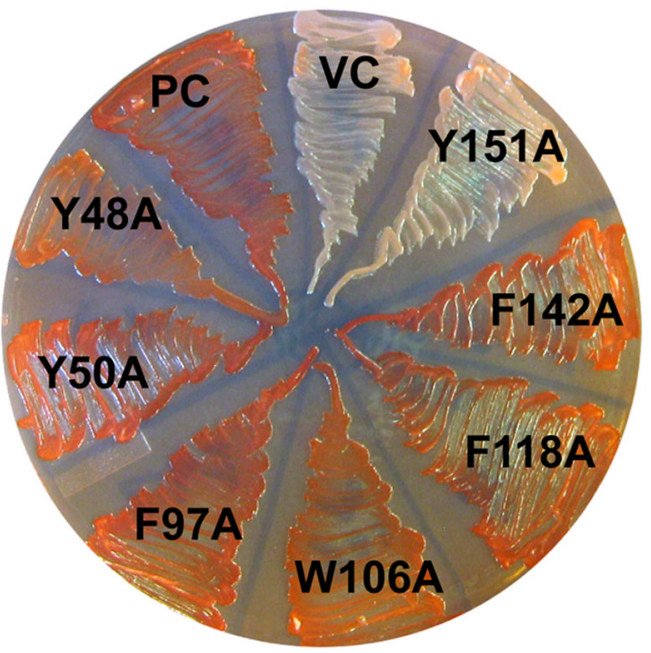

(c)

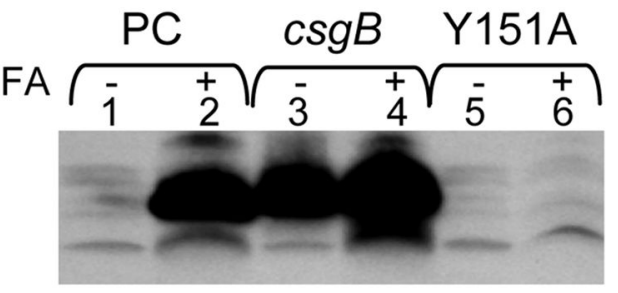

(d)

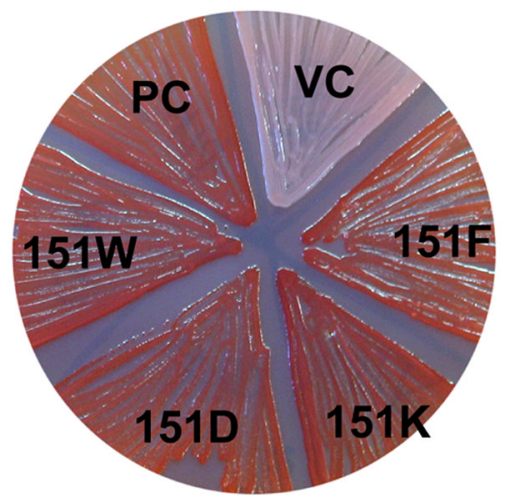

(b) PC VC csgB Y48A Y50A F97A W106AF118A F142A Y151A

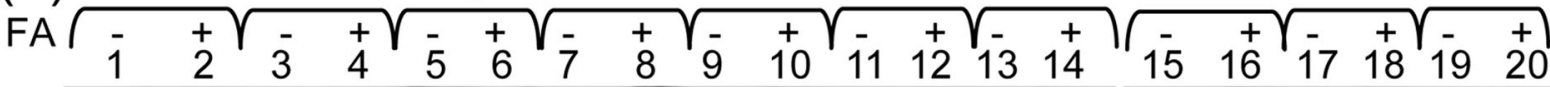

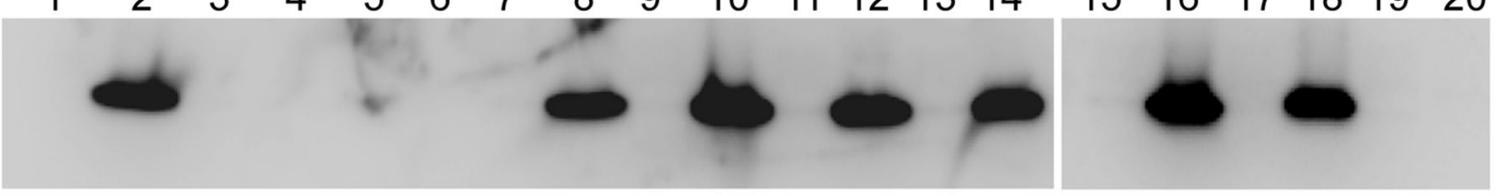

Figure 3. Ala scan mutagenesis of aromatic residues in $\mathrm{CsgA}$ amyloid core regions (a) CR-YESCA plate with $\operatorname{csg} A$ cells transformed with the plasmids encoding $\mathrm{CsgA}$, $\mathrm{CsgA}^{\mathrm{Y} 48 \mathrm{~A}}, \mathrm{Csg}^{\mathrm{Y} 50 \mathrm{~A}}, \mathrm{Csg}^{\mathrm{F} 97 \mathrm{~A}}, \mathrm{Csg}^{\mathrm{W} 106 \mathrm{~A}}, \mathrm{Csg}^{\mathrm{F} 118 \mathrm{~A}}, \mathrm{CsgA}^{\mathrm{F} 142 \mathrm{~A}}$ or $\operatorname{CsgA}^{\mathrm{Y} 151 \mathrm{~A}}$. (b)

Western blots of whole-cell lysates from $\operatorname{csg} B$ cells and $\operatorname{csg} A$ cells containing plasmids encoding wild-type CsgA or indicated Ala mutants. (c) Western blot of whole-cells and underlying agar (agar plugs) from $\operatorname{csg} B$ cells and $\operatorname{csg} A$ strains containing plasmids encoding CsgA or CsgA Y151A (d) The CR-YESCA plate with $\operatorname{csgA}$ cells transformed with the plasmids encoding CsgA, CsgA ${ }^{\text {Y151F }}, \operatorname{CsgA}^{\text {Y151W }}, \operatorname{Csg}^{\text {F151D }}$ or CsgA ${ }^{\text {Y151K. }}$. 
(a)

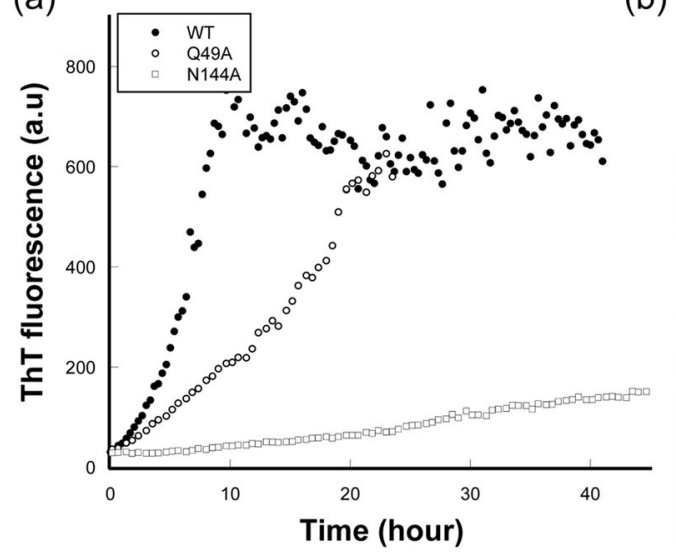

(c)

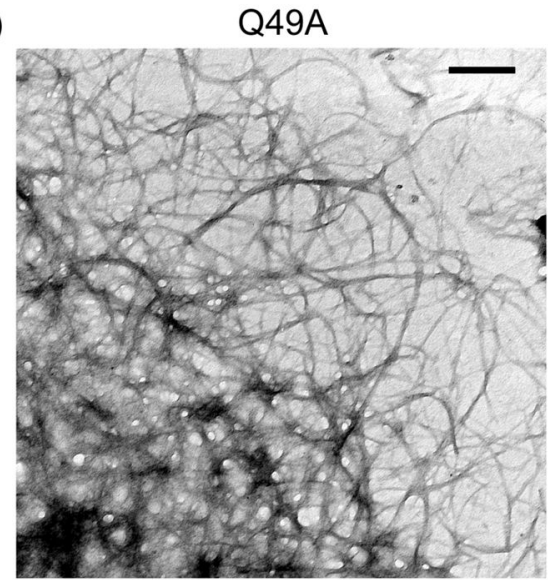

(b)

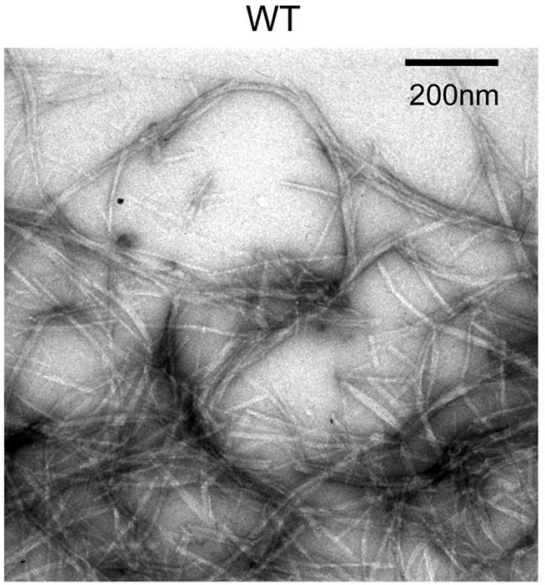

(d)

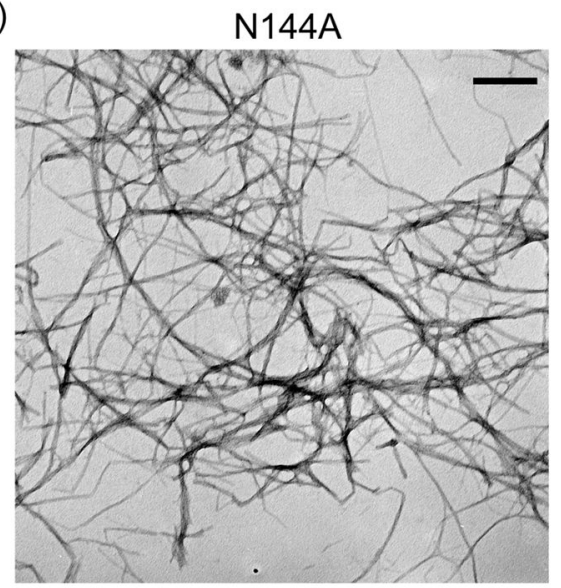

Figure 4. In vitro self-polymerization of $\mathrm{CsgA}^{\mathrm{Q49A}}$ and $\mathrm{CsgA}^{\mathrm{N} 144 \mathrm{~A}}$ are defective

(a) The fluorescence of $40 \mu \mathrm{M} \operatorname{CsgA}$ (solid circle), CsgA $\mathrm{Q}^{49 \mathrm{~A}}$ (open circle) and $\mathrm{Csg}^{\mathrm{N} 144 \mathrm{~A}}$ (open square) mixed with $20 \mu \mathrm{M}$ ThT was measured in 10-minute intervals at $495 \mathrm{~nm}$ after excitation at $438 \mathrm{~nm}$. a.u., arbitrary units. (b-d) Negative-stain EM micrographs of in vitro selfpolymerized fibers of $\operatorname{Csg} \mathrm{A}(\mathrm{b}), \mathrm{CsgA}^{\mathrm{Q} 49 \mathrm{~A}}$ (c) and $\operatorname{Csg} \mathrm{A}^{\mathrm{N} 144 \mathrm{~A}}$ (d). Scale bars are equal to 200 $\mathrm{nm}$. 
(a) Soluble CsgA-like molecules
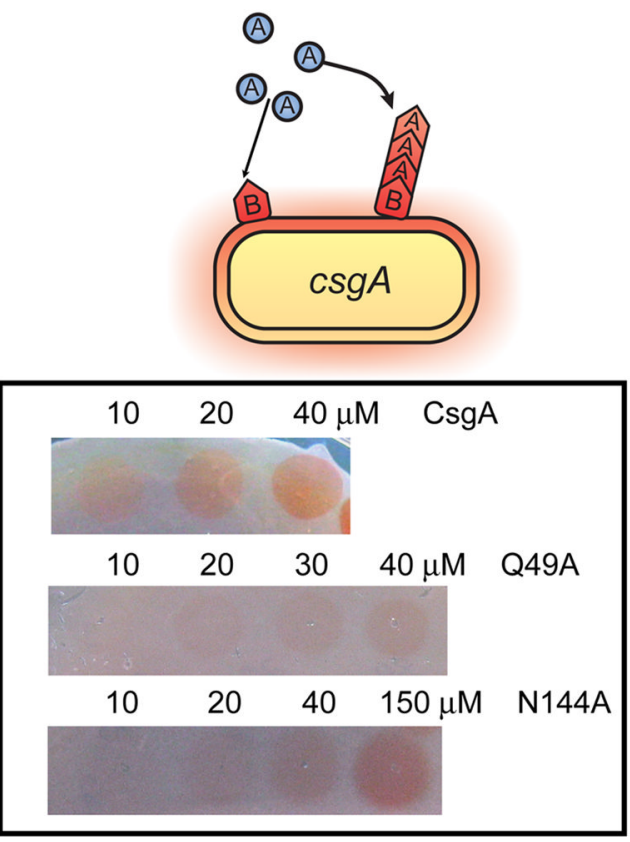

(b)
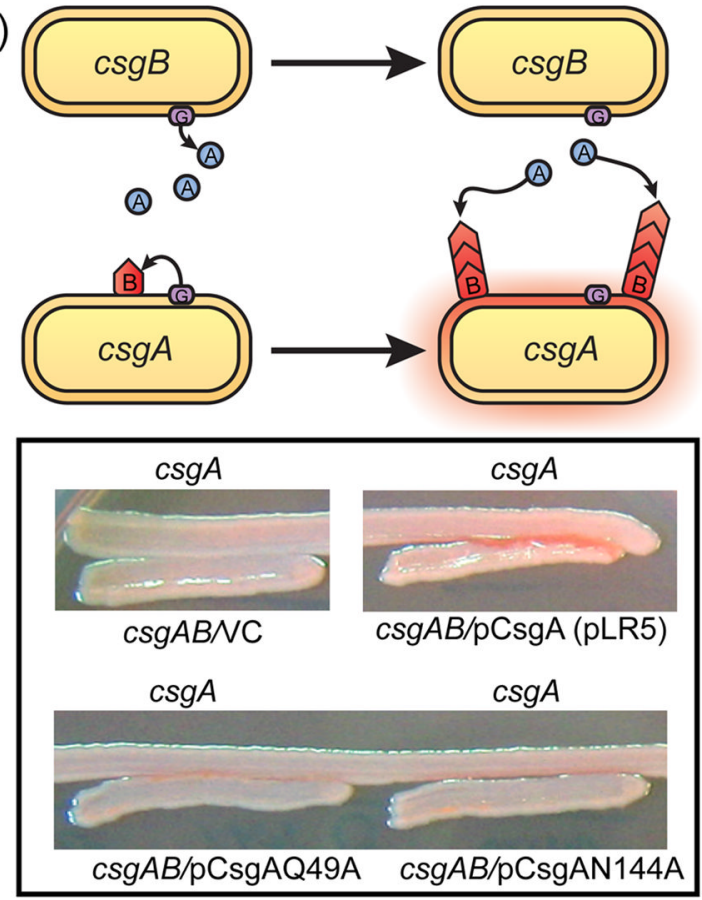

Figure 5. $\mathrm{CsgA}^{\mathrm{Q49A}}$ and $\mathrm{Csg}^{\mathrm{N144A}}$ are defective in response to CsgB-mediated heteronucleation (a) A schematic presentation of the overlay assay in which soluble CsgA is spotted onto cells $(\operatorname{csg} A)$ expressing the nucleator protein, $\mathrm{CsgB}$, where $\mathrm{Csg}$ A polymerization is catalyzed by CsgB (top). Freshly purified CsgA, $\operatorname{Csg}^{\mathrm{Q}} 49 \mathrm{~A}$ or $\mathrm{Csg}^{\mathrm{N} 144 \mathrm{~A}}$ at the indicated concentrations was spotted onto on $\operatorname{csg} A$ cells. After $10 \mathrm{~min}$ incubation, cells were stained with $0.5 \mathrm{mg} / \mathrm{ml}$ Congo red solution and washed by potassium phosphate buffer (bottom). (b) A schematic presentation of the interbacterial complementation assay in which soluble CsgA molecules secreted from $\operatorname{csg} B$ cells interact with CsgB on the surface of $c s g A$ cells to form fibers (top). $\operatorname{csg} B A$ cells containing plasmids encoding $\mathrm{Csg} \mathrm{A}, \mathrm{Csg} \mathrm{A}^{\mathrm{Q} 49 \mathrm{~A}}$ or $\mathrm{Csg}^{\mathrm{N} 144 \mathrm{~A}}$ were grown adjacently to $c s g A$ cells on a CR-YESCA plate for $48 \mathrm{~h}$ at $26^{\circ} \mathrm{C}$ (bottom). 

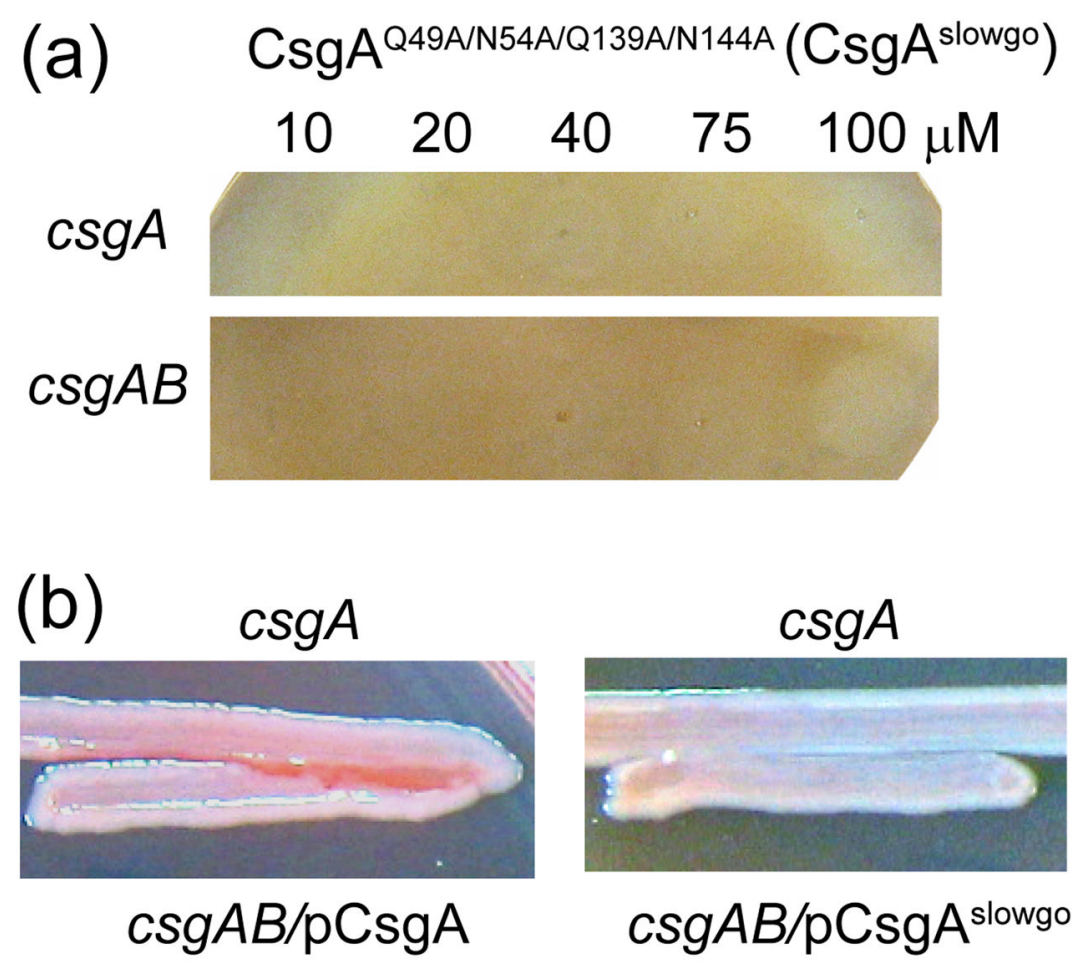

Figure 6. The heteronucleation responsiveness of $\mathrm{CsgA}^{\text {slowgo }}\left(\mathrm{CsgA}^{\mathrm{Q49A} / N 54 A / Q 139 A / N 144 A}\right)$ (a) Freshly purified homogeneous $\mathrm{CsgA}^{\text {slowgo }}$ at the indicated concentrations was spotted onto $\operatorname{csg} A$ or $\operatorname{csg} B A$ cells. After $10 \mathrm{~min}$ incubation, cells were stained with $0.5 \mathrm{mg} / \mathrm{ml}$ Congo red solution and washed by potassium phosphate buffer. (b) $\operatorname{csg} B A$ cells containing plasmids encoding CsgA or CsgA ${ }^{\text {slowgo }}$ were grown adjacently to $\operatorname{csg} A$ cells on a CR-YESCA plate for $48 \mathrm{~h}$ at $26^{\circ} \mathrm{C}$. 

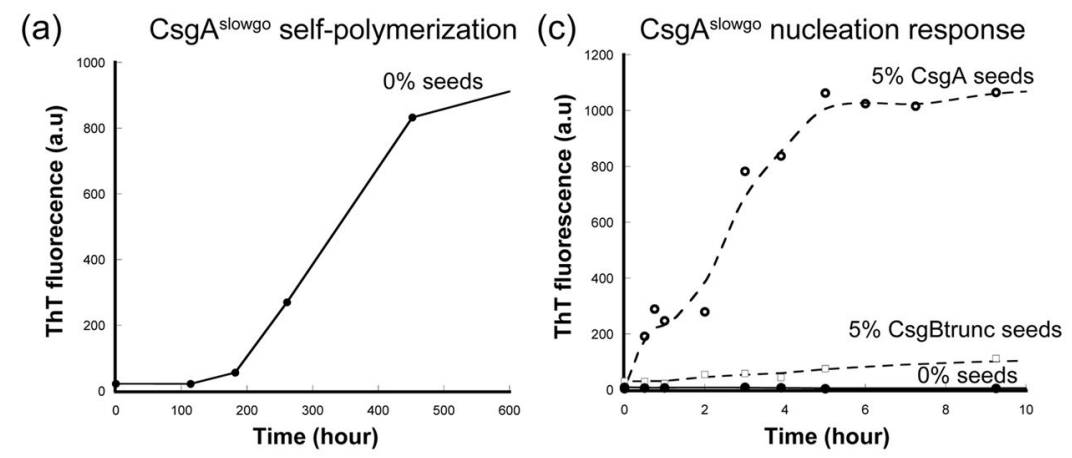

(b)

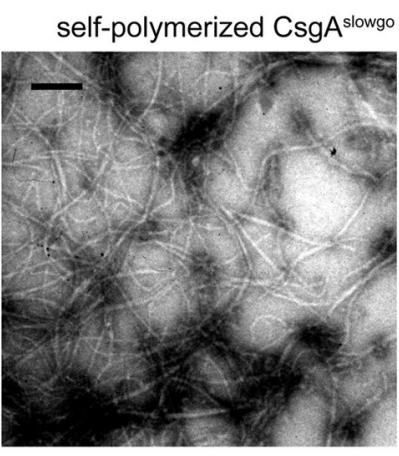

(d)
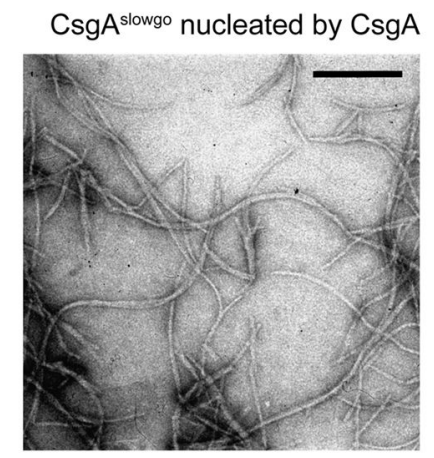

Figure 7. In vitro self-polymerization and seeding responsiveness of $\operatorname{Csg}^{\text {slowgo }}$

(a) ThT fluorescence intensity of $40 \mu \mathrm{M} C \operatorname{csg} \mathrm{A}^{\text {slowgo }}$ incubated for the indicated time intervals. a.u., arbitrary units. (b) Negative stain TEM micrograph of in vitro self-polymerized aggregates of $\mathrm{CsgA}^{\text {slowgo }}$. Scale bars are equal to $200 \mathrm{~nm}$. (C) $5 \% \mathrm{CsgA}$ seeds, $\mathrm{CsgB}_{\text {trunc }}$ seeds by weight or buffer vehicle were added to $60 \mu \mathrm{M} \mathrm{Csg} \mathrm{A}^{\text {slowgo }}$ and the polymerization was measured by ThT fluorescence assay. The fluorescence intensity of nucleated Csg $\mathrm{A}^{\text {slowgo }}$ samples were shown at the indicated time intervals. The contribution to fluorescence intensity by seeds was subtracted. (d) Negative-stain TEM micrograph of in vitro polymerized aggregates of $\mathrm{Csg}^{\text {slowgo }}$ promoted by CsgA. Scale bars are equal to $200 \mathrm{~nm}$. 
(a)

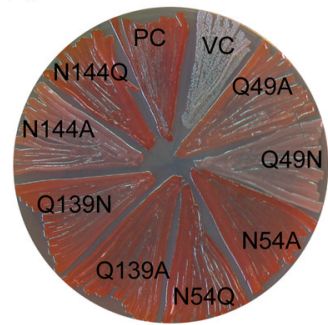

(b)

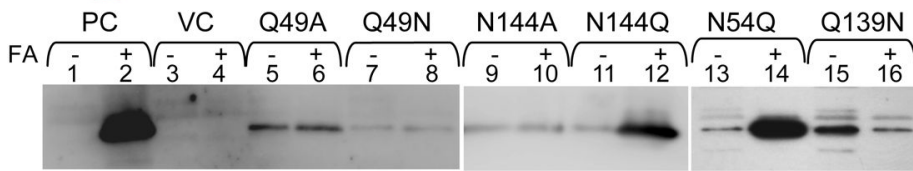

(c)

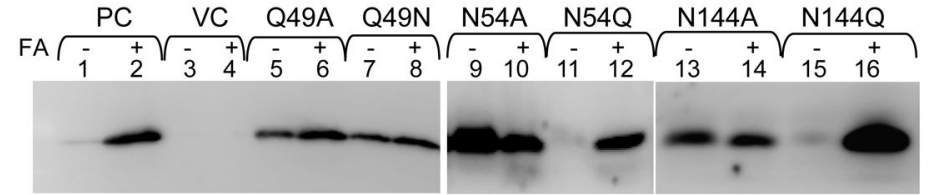

(e)

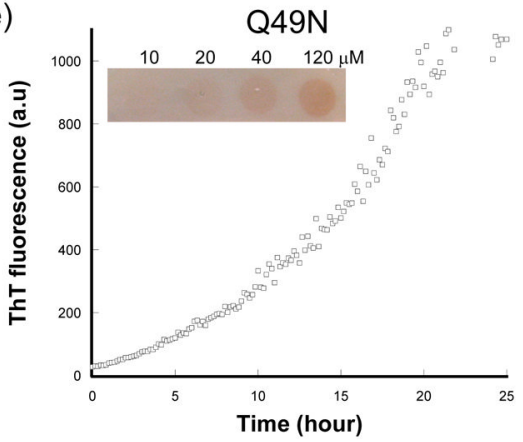

(d)

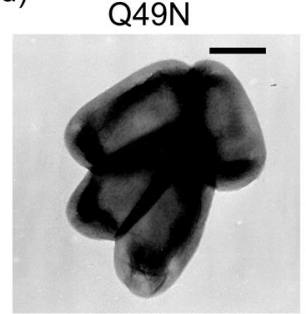

N144Q

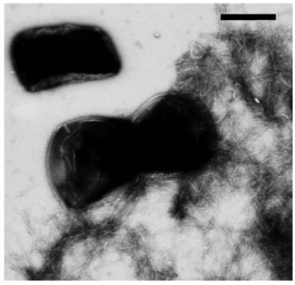

Figure 8. Conservative $Q / \mathrm{N}$ substitutions at positions 49, 54, 139 and 144

(a) CR-YESCA plate with $\operatorname{csg} A$ transformed with the plasmids encoding $\operatorname{CsgA}, \operatorname{CsgA} \mathrm{Q}^{\mathrm{A} 9 \mathrm{~A}}$, $\mathrm{CsgA}^{\mathrm{Q} 49 \mathrm{~N}}, \mathrm{CsgA}^{\mathrm{N} 54 \mathrm{~A}}, \mathrm{Csg}^{\mathrm{N} 54 \mathrm{Q}}, \mathrm{CsgA}^{\mathrm{Q} 139 \mathrm{~A}}, \mathrm{Csg}^{\mathrm{Q} 139 \mathrm{~N}}, \operatorname{Csg}^{\mathrm{N} 144 \mathrm{~A}}$ or $\operatorname{Csg}^{\mathrm{N} 144 \mathrm{Q}}$.

(b) Western blots of whole-cell lysates from $\operatorname{csg} A$ cells containing plasmids encoding wildtype $\operatorname{Csg} A, C s g A^{\mathrm{Q} 49 \mathrm{~A}}, \mathrm{CsgA}^{\mathrm{Q} 49 \mathrm{~N}}, \mathrm{CsgA}^{\mathrm{N} 54 \mathrm{Q}}, \mathrm{CsgA}^{\mathrm{Q} 139 \mathrm{~N}}, \operatorname{Csg}^{\mathrm{N} 144 \mathrm{~A}}$ or $\operatorname{Csg}^{\mathrm{N} 144 \mathrm{Q}}$. (c) Western blot of whole-cells and underlying agar (agar plugs) from $\operatorname{csg} A$ strains containing plasmids encoding CsgA, CsgA ${ }^{\mathrm{Q} 49 \mathrm{~A}}, \operatorname{CsgA}^{\mathrm{Q} 49 \mathrm{~N}}, \operatorname{CsgA}^{\mathrm{N} 54 \mathrm{~A}}, \operatorname{CsgA}^{\mathrm{N} 54 \mathrm{Q}}, \operatorname{CsgA}^{\mathrm{N} 144 \mathrm{~A}}$ or $\mathrm{CsgA}^{\mathrm{N} 144 \mathrm{Q}}$. Samples were treated with (+) or without (-) FA. The blots were probed with antiCsgA antibody. (d) Negative-stain TEM micrographs of $\operatorname{csg} A$ cells containing plasmids encoding CsgA ${ }^{\mathrm{Q} 49 \mathrm{~N}}$ and $\mathrm{Csg}^{\mathrm{N} 144 \mathrm{Q}}$. Scale bars are equal to $500 \mathrm{~nm}$. (e) The polymerization of $56 \mu \mathrm{M} \operatorname{CsgA}^{\mathrm{Q} 49 \mathrm{~N}}$ was measured by ThT fluorescence. Congo red staining of $\operatorname{csgA}$ cells overlaid with $\operatorname{CsgA} \mathrm{Q}^{\mathrm{Q} 9 \mathrm{~N}}$ at various concentrations is shown in the graph inset. (f) CR-YESCA plate of a $\operatorname{csg} A$ strain transformed with the control vector pLR2 or the plasmids encoding CsgA, $\operatorname{Csg} \mathrm{A}^{\mathrm{Q} 49 \mathrm{~N}}$ or $\operatorname{Csg} \mathrm{A}^{\mathrm{Q} 49 \mathrm{~N} / \mathrm{N} 144 \mathrm{Q}}$. 